\title{
Architektur für die kooperative Auftragsabwicklung
}

\author{
Dimitrios Gizanis, Christine Legner, Hubert Österle \\ Universität St. Gallen
}

\begin{abstract}
Zusammenfassung: Das Aufbrechen traditioneller Wertschöpfungsketten führt dazu, dass an der Abwicklung eines Kundenauftrags oft mehrere Partner beteiligt sind. Während mit der Implementierung von ERP-Systemen durchgängige interne Prozesse geschaffen wurden, existieren an den Schnittstellen zu externen Geschäftspartnern zahlreiche Ineffizienzen. Typische Beispiele sind die manuelle Wiedereingabe von Auftragsdaten, fehlende bzw. unvollständig weitergeleitete Informationen und Pufferbestände. Diese wirken sich einerseits auf die Kosten, andererseits auf Durchlaufzeiten, Service Level und damit auf die Kundenzufriedenheit aus. Verbesserungen in der überbetrieblichen bzw. kooperativen Auftragsabwicklung setzen abgestimmte Prozesse und Daten mit Partnern und Kunden sowie flexible Informationssystemarchitekturen voraus. Der Beitrag entwickelt eine Architektur für die kooperative Auftragsabwicklung auf den Gestaltungsebenen der Strategie, der Geschäftsprozesse und der Informationssysteme.
\end{abstract}

Schlüsselworte: Auftragsabwicklung, Architektur, Wertschöpfungsnetzwerke, Kooperationsprozesse, Grenzen von ERP-Systemen, Integration

\section{Einleitung}

\subsection{Trends in der Auftragsabwicklung}

Die Auftragsabwicklung ist ein operativer Kernprozess jedes Unternehmens. Mit massiven Investitionen zur Einführung von ERP-Systemen haben Unternehmen in den letzten Jahren durchgängige interne Prozesse in der Auftragsabwicklung geschaffen und sind damit wettbewerbsfähig geblieben.

Der Wandel vom Verkäufer- zum Käufermarkt und die Globalisierung im Sinne einer physischen Disintegration verlagern den Wettbewerb zunehmend auf Wertschöpfungsnetzwerke [ChKu00, S. 268; Flei01, S. 18ff]. Wettbewerbsvorteile resultieren dabei aus der Strukturierung der Wertschöpfungskette und der Konfiguration, Transaktionsform und Koordination der Wertschöpfungsprozesse [Zent $\left.{ }^{+} 04,218 \mathrm{ff}\right]$. 
Die organisatorischen Grenzen setzen sehr häufig auch die Grenzen durchgängiger Informationsflüsse. Bei der überbetrieblichen Auftragsabwicklung fehlen diese heute in der Regel. Befragungen von Entscheidungsträgern in Europa und USA haben einen geringen Integrationsgrad von externen Partnern in der Auftragsabwicklung von Unternehmen ergeben [John03; Nerv02, S. 8ff]. Als Folge bestehen an den organisationsübergreifenden Schnittstellen zu externen Partnern, Kunden oder anderen Geschäftsbereichen Ineffizienzen, wie z. B. hohe Durchlaufzeiten und Bestände, unzuverlässige Verfügbarkeitsprüfungen und mangelnde Steuerungsmöglichkeiten. Allerdings planen die befragten Unternehmen, Kooperationen in der Auftragsabwicklung zu intensivieren und Ausführungsprozesse u. a. in den Bereichen Produktion und Distribution an Partner abzugeben.

\subsection{Die kooperative Auftragsabwicklung}

Die Kundenauftragsbearbeitung umfasst sämtliche betriebliche Aktivitäten von der Erfassung eines Auftrags über den Warentransport bis hin zur Verrechnung der Leistung an den Kunden und dessen Bezahlung [Otto04, S. 14ff; KuHe03, S. 645]. Als kooperative Auftragsabwicklung verstehen wir eine durchgängige Auftragsbearbeitung verschiedener interner und externer Partner bei der Erfüllung eines Kundenauftrags. Dabei unterscheidet sich der Prozessfluss von der klassischen Auftragsabwicklung in den Aufgaben, in die Partner miteinbezogen werden. Dies betrifft im Allgemeinen die Beschaffung (Partnerbestimmung), die Transport- und Rechnungsabwicklung (Koordination der Lieferung und Fakturierung über mehrere Partner) sowie das Beschwerdemanagement (Koordination der Reklamationsbearbeitung).

Beispiele für die kooperative Auftragsabwicklung sind insbesondere in der Hightech-Industrie zu finden. So hat Dell konsequent einen Direktvertrieb und eine Auftragsfertigung mit Partnern etabliert [Krae ${ }^{+} 00$, S. 8f]. Abgestimmte Prozesse mit Kunden und Lieferanten begründen die geringen Durchlaufzeiten und Lagerbestände bei Dell [Rock00; Schr02].

\subsection{Architektur für die kooperative Auftragsabwicklung}

Architekturen stellen Gestaltungsmodelle dar, die Bausteine einer Lösung in $\mathrm{Zu}-$ sammenhang stellen [Nola97, S. 124; Sinz97, S. 875; Stru97, S. 35]. Ihre Entwicklung gilt als eine der Hauptaufgaben der Wirtschaftsinformatik [Seib90, S. 14]. Während Architekturen im innerbetrieblichen Bereich insbesondere in Zusammenhang mit der Einführung von ERP-Systemen entstanden sind, fehlt es an systematischen, anbieterunabhängigen Modellen für überbetriebliche Prozesse [Alt04, S. 12].

Die hier vorgeschlagene Architektur für die kooperative Auftragsabwicklung entwickelt Gestaltungshilfen für den überbetrieblichen Auftragsdurchlauf. Sie 
berücksichtigt hierbei die Ebenen des Business Engineering (Strategie, Prozesse, Informationssysteme) [Öste95, S. 16ff] und baut auf der generischen Architektur des Echtzeitunternehmens von [Alt04, S. 122ff] auf (s. Abbildung 1).
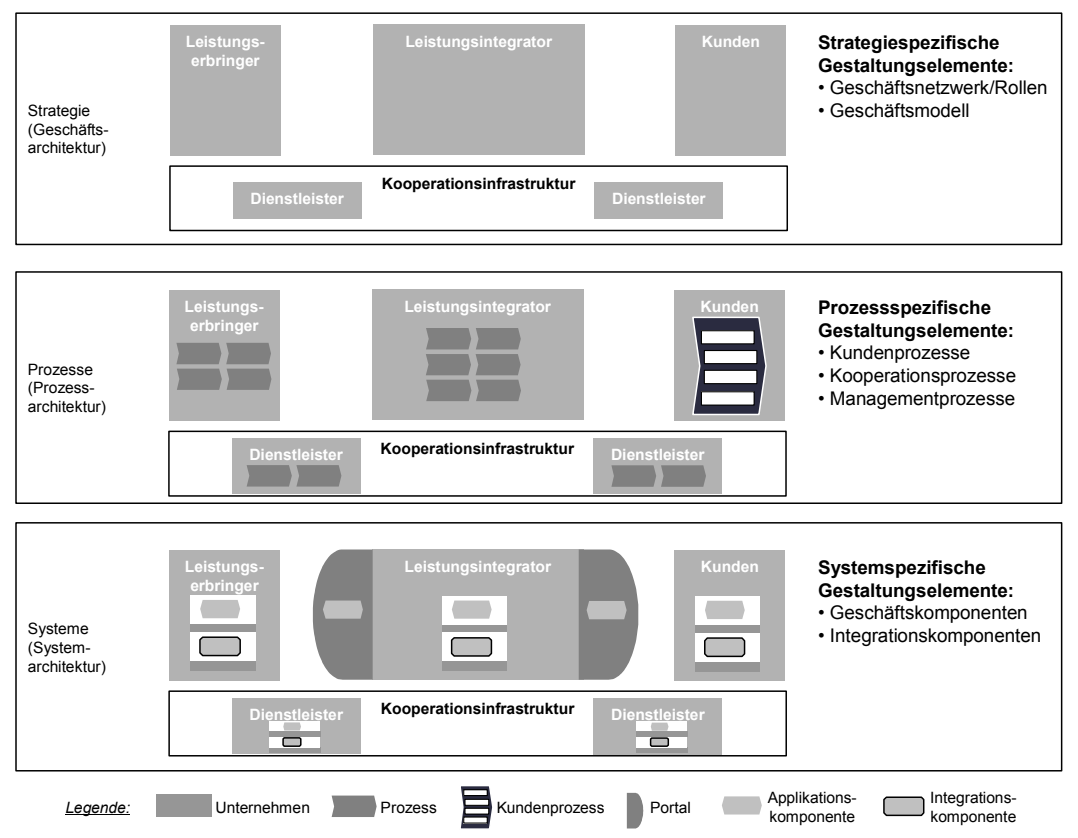

Abbildung 1: Architektur des Echtzeitunternehmens nach [Alt04, S. 122ff]

Die Quellen für die Ausprägung der hier vorgeschlagenen Architektur sind:

- Wissenschaftliche und anwendungsorientierte Literatur zur (kooperativen) Auftragsabwicklung [Mert97; Mert ${ }^{+}$99; Sche92; Sche02; KuHe03; BuZe04, S. 167; Otto04; Röhr ${ }^{+} 00$, S. 183ff]

- Vier Fallstudien zur kooperativen Auftragsabwicklung aus unterschiedlichen Branchen [Giza ${ }^{+}$03, S. 545ff; Seng03; GiLe04, S. 69ff; GiHe04]

- Spezifische Lösungen von Softwareanbietern für die kooperative Auftragsabwicklung [Newt01; Alt $\left.{ }^{+} 04\right]$

\section{Geschäftsarchitektur}

Die Geschäftsarchitektur, häufig auch als Geschäftsmodell bezeichnet, ist das Ergebnis der Strategiedefinition eines Unternehmens und setzt die Grundlagen für die Gestaltung von Prozessen und Systemen. Die Geschäftsarchitektur umfasst 
den für den Kunden generierten Nutzen im Sinne einer „Value Proposition“, das Ertragsmodell und die an der Wertschöpfung beteiligten Partner. Im Zusammenhang mit der kooperativen Auftragsabwicklung steht im Vordergrund, welcher Zusatznutzen durch den Einbezug von Partnern entsteht, wie sich das Geschäftsnetzwerk dadurch verändert und welche Rollen die Partner einnehmen.

\subsection{Geschäftsnetzwerk und Rollen}

Charakteristisch für die kooperative Auftragsabwicklung sind Leistungsintegratoren, die neben der eigentlichen Leistungserstellung für den Kunden verschiedene Leistungserbringer koordinieren. Das Geschäft dieser auch als „Orchestrators“ oder „Aggregators“ bezeichneten Leistungsintegratoren ist nicht der Verkauf einzelner Marktleistungen, sondern analog zu klassischen Handelsfunktionen die Leistungsvermittlung [Hint03, S. 615ff; Zent04+, 218ff].

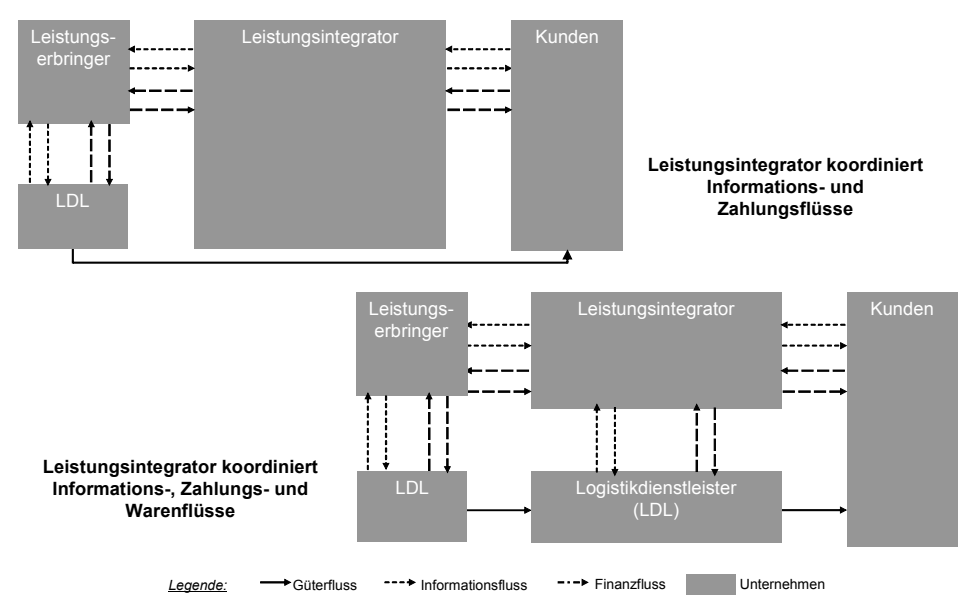

Abbildung 2: Typen von Leistungsintegratoren ${ }^{1}$

Je nach Koordinationstiefe sind zwei Typen von Leistungsintegratoren zu unterscheiden (s. Abbildung 2):

- Der Leistungsintegrator koordiniert lediglich die Informations- und Zahlungsflüsse (die Waren werden von den Lieferanten im Streckengeschäft direkt an die Kunden geschickt). Beispiel: Lekkerland (Schweiz) bietet Lebensmittel und Waren für den Convenience-Markt der Schweiz an. Mit der Positio-

1 Der Logistikdienstleister (LDL) wird aufgrund seiner wichtigen Rolle bei der Koordination von Materialflüssen zusätzlich zu den Leistungserbringern aufgeführt. 
nierung als Leistungsintegrator verfolgt Lekkerland das Ziel, seine Kunden stärker an sich zu binden. Ein verbessertes Leistungsangebot soll Tankstellenbetreibern ermöglichen, ihr komplettes Sortiment zentral bei Lekkerland zu bestellen, anstatt Teilsortimente über verschiedene Lieferanten (Leistungserbringer) und deren heterogenes Bestellwesen zu beschaffen. Hierdurch soll sich der administrative Aufwand für die Bestell- und Rechnungsabwicklung der Tankstellenbetreiber deutlich reduzieren. Lekkerland koppelt hierfür Vertragslieferanten an die eigene Plattform, wodurch diese von einer stärkeren Automatisierung profitieren sollen [Giza ${ }^{+} 03$, S. 545ff].

- Der Leistungsintegrator koordiniert sowohl Informations-, Zahlungs- als auch Materialflüsse. Beispiel: Dell steuert als Leistungsintegrator die gesamte Supply Chain selbst, indem Kundenaufträge automatisch Bestellungen bei Lieferanten und Herstellern auslösen, die dann mit Hilfe von Logistikdienstleistern (LDL) über Distributions- und Montagepunkte konsolidiert und an den Kunden geliefert werden [Krae ${ }^{+} 00$, S. 8ff].

\begin{tabular}{|c|c|c|}
\hline Rolle & Nutzenpotenziale & Risiken \\
\hline Kunde & $\begin{array}{l}\text { - Reduktion der Beschaffungsquellen / -komplexität } \\
\text { (Effizientere Beschaffungsabwicklung durch weniger } \\
\text { Rechnungen und Zahlungsvorgänge) } \\
\text { - Verringerung von Beständen } \\
\text { - Gebündelte Informationen über Produktangebote und } \\
\text { deren Verfügbarkeit, aktuelle Auftragsstatus etc. }\end{array}$ & $\begin{array}{l}\text { - Eingeschränkte } \\
\text { Beschaffungs- } \\
\text { freiheit }\end{array}$ \\
\hline $\begin{array}{l}\text { Leistungs- } \\
\text { integrator }\end{array}$ & $\begin{array}{l}\text { - Stärkere Kundenbindung aufgrund einer zentralen } \\
\text { Kundenansprache („One-face-to-the-customer“) und } \\
\text { integrierter Prozesse } \\
\text { - Höhere Umsätze durch Erweiterung des Leistungs- } \\
\text { angebots mit komplementären Produkten und } \\
\text { Services (Cross- und Up-Selling) } \\
\text { - Höhere Flexibilität bei der Bestimmung von Partnern } \\
\text { zur effizienten Erfüllung eines Kundenauftrags auf } \\
\text { Basis übergreifender Echtzeitinformationen } \\
\text { - Bessere Planungs- und Steuerungsmöglichkeiten im } \\
\text { Netzwerk }\end{array}$ & $\begin{array}{l}\text { - Zusatzaufwand } \\
\text { für die Koor- } \\
\text { dination der } \\
\text { Leistungs- } \\
\text { erbringer } \\
\text { - Grösster Image- } \\
\text { verlust bei } \\
\text { Problemen }\end{array}$ \\
\hline $\begin{array}{l}\text { Leistungs- } \\
\text { erbringer } \\
\text { (inkl. } \\
\text { LDL) }\end{array}$ & $\begin{array}{l}\text { - Reduktion des Administrationsaufwands (zentraler } \\
\text { Partner) } \\
\text { - Bessere Kapazitätsplanung aufgrund zeitnaher } \\
\text { Auftragsinformationen } \\
\text { - Spezialisierung/Konzentration auf Kernkompetenzen }\end{array}$ & $\begin{array}{l}\text { - Verlust des } \\
\text { direkten Kun- } \\
\text { denkontakts } \\
\text { - Offenlegung } \\
\text { sensibler Daten } \\
\text { - Austauschbar- } \\
\text { keit }\end{array}$ \\
\hline
\end{tabular}

Tabelle 1: Nutzenpotenziale und Risiken in der kooperativen Auftragsabwicklung

In beiden Fällen übernimmt der Leistungsintegrator im Vergleich zur klassischen Auftragsabwicklung zusätzliche koordinative Aufgaben für den Kunden, wie z. B. 
die Weiterleitung der Auftragsdaten an Lieferanten, die Transportkoordination oder die Leistungsverrechnung. Die dadurch entstehenden Nutzenpotenziale und Risiken fasst Tabelle 1 für die beteiligten Partner zusammen.

\subsection{Geschäftstreiber für die kooperative Auftragsabwicklung}

Die Geschäftstreiber fassen wichtige Einflussfaktoren zusammen, die zur Entwicklung des Geschäftsmodells der kooperativen Auftragsabwicklung führen. Es lassen sich einerseits kostenorientierte, andererseits qualitäts- und damit umsatzorientierte Treiber unterscheiden (s. Tabelle 2 und [Huan04, S. 1]).

\begin{tabular}{|c|c|}
\hline \multirow{2}{*}{ : } & $\begin{array}{l}\text { Konzentration auf Kernkompetenzen. Unternehmen übertragen einzelne } \\
\text { Prozessteile oder Kernleistungen an externe Partner [Heus99, S. 36ff; Marb00, S. } \\
\text { 5]. Dabei reichen die Gründe von einem kostengetriebenen Fremdbezug bis hin zu } \\
\text { einer strategischen Partnerschaft [KaKa00]. Outsourcing-Aktivitäten führen zu } \\
\text { engeren Abstimmungen in der überbetrieblichen Auftragsabwicklung. }\end{array}$ \\
\hline & $\begin{array}{l}\text { Verbesserung der operativen Effizienz. Ein überbetrieblicher Auftragsdurchlauf } \\
\text { verbindet die Datenentstehung beim Kunden mit der konsolidierten Bereitstellung } \\
\text { der Auftragsdaten bei den verschiedenen Adressaten. Durch den schnellen (durch- } \\
\text { gängigen) Austausch von Auftragsdaten kann die Effizienz zusammenhängender } \\
\text { Prozesse wie Produktionsplanung und Lagerhaltung gesteigert werden [Seng03]. }\end{array}$ \\
\hline \multirow{2}{*}{ 这 } & $\begin{array}{l}\text { Verbesserung der Kundenbeziehung. Kundennutzen entsteht dur } \\
\text { sierung des Leistungsangebots oder aufgrund einer Komplexitäts- } \\
\text { reduktion in der Beschaffung. In der Automobil- oder Bauindustri } \\
\text { beispielsweise Systemlieferanten bzw. Generalunternehmer versch } \\
\text { Leistungserbringer für ihre Kunden [Mätz96, S. 71ff; WeSc03, S. } 33\end{array}$ \\
\hline & $\begin{array}{l}\text { Erweiterung des Leistungsangebots. Die Erweiterung der Produktpalette eines } \\
\text { Unternehmens um komplementäre Produkte und Dienstleistungen (auch von } \\
\text { Dritten) ist die Basis für höhere Umsätze und schafft eine Differenzierung gegen- } \\
\text { über Wettbewerbern [WiBa99, S. 139; O1Ka03, S. 162]. Das Internet-Handels- } \\
\text { unternehmen myToys.de ergänzt beispielsweise sein Sortiment durch Artikel (z. B. } \\
\text { Kindermöbel) die es selbst nicht im eigenen Bestand führt [Müll04 S. 59ff]. }\end{array}$ \\
\hline
\end{tabular}

Tabelle 2: Geschäftstreiber für die kooperative Auftragsabwicklung

\section{Prozessarchitektur}

Die kooperative Auftragsabwicklung bricht die heute dominanten, unternehmensintern integrierten Prozesse auf und verteilt die Aufgaben zwischen den Teilnehmern der Wertschöpfungskette neu. Sie beginnt mit dem Kundenprozess, also dem Prozess des Kunden, und definiert dadurch das Angebot des Leistungsintegrators sowie den Informations-, den Waren- und den Finanzfluss und nicht zuletzt die Managementprozesse. 


\subsection{Kundenprozesse in der Beschaffung}

Ausgangspunkt für die Gestaltung der kooperativen Auftragsabwicklung sind die beschaffungsbezogenen Prozesse des Kunden. Der Leistungsintegrator schafft Kundennutzen, indem er zusätzliche Leistungen anbietet, die den Kundenprozess besser abdecken. Dabei übernimmt er vor allem Koordinationsaufgaben für den Kunden. Diese Leistungen betreffen die Disposition, Bestell- und Rechnungsabwicklung. Dem Warenfluss vorauseilende Lieferinformationen (z. B. Advanced Shipping Notes, ASN) können beispielsweise die Planungsvorgänge des Kunden verbessern und dabei ein rechtzeitiges Handeln einleiten, z. B. bei Voranzeige einer verspäteten Auslieferung.

\begin{tabular}{|c|c|c|}
\hline Aufgaben & Typische Probleme des Kunden & Mögliche Zusatzleistungen \\
\hline $\begin{array}{l}\text { Bedarf } \\
\text { spezifizieren }\end{array}$ & $\begin{array}{ll}\text { - } & \text { Falsche Planung } \\
\text { - } & \text { Hohe Sicherheitsbestände } \\
\text { - } & \text { Mangelnde Kenntnis des } \\
& \text { Angebots }\end{array}$ & $\begin{array}{l}\text { Bewirtschaftung des } \\
\text { Kundenlagers } \\
\text { - Alternative } \\
\text { Produktvorschläge }\end{array}$ \\
\hline $\begin{array}{l}\text { Lieferant } \\
\text { auswählen }\end{array}$ & $\begin{array}{l}\text { Pflege mehrerer Geschäftsbe- } \\
\text { ziehungen und Betrieb einer } \\
\text { komplexeren Infrastruktur } \\
\text { Kein effizientes Sourcing, z. B. } \\
\text { hinsichtlich Preis, Verfügbarkeit }\end{array}$ & 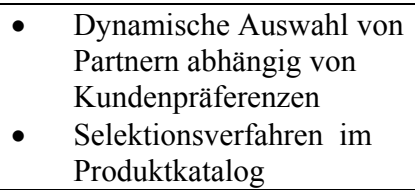 \\
\hline $\begin{array}{l}\text { Bestellung } \\
\text { erteilen }\end{array}$ & $\begin{array}{l}\text { - Hoher Aufwand für die Erteilung } \\
\text { und Überwachung mehrerer } \\
\text { Bestellungen } \\
\text { - } \quad \text { Disposition mehrerer Transporte }\end{array}$ & $\begin{array}{ll}\text { - } & \text { Konsolidierte Bestellung } \\
\text { - } & \text { Konsolidierte } \\
\text { - } & \text { Auftragsbestätigung } \\
\text { Verbindliche } \\
\text { Terminzusagen }\end{array}$ \\
\hline $\begin{array}{l}\text { Status } \\
\text { verfolgen }\end{array}$ & $\begin{array}{ll}\text { - } & \text { Unzureichende Transparenz } \\
\text { - } & \text { Koordinationsaufwand bei } \\
& \text { Statusanfragen }\end{array}$ & $\begin{array}{ll}\text { - } & \text { Konsolidierte } \\
\text { Statusinformationen } \\
\text { - } & \text { Proaktive Informationen, } \\
\text { z. B. bei Verspätungen } \\
\end{array}$ \\
\hline $\begin{array}{l}\text { Leistung } \\
\text { empfangen } \\
\text { und nutzen }\end{array}$ & $\begin{array}{ll}- & \text { Unsicherheit bez. Warenankunft } \\
\text { - } & \text { Hoher Aufwand am Warenein- } \\
\text { gang durch mehrere Transporte }\end{array}$ & $\begin{array}{ll}- & \text { Lieferavis } \\
\bullet & \text { Konsolidierte Lieferungen }\end{array}$ \\
\hline $\begin{array}{l}\text { Rechnung } \\
\text { prüfen }\end{array}$ & 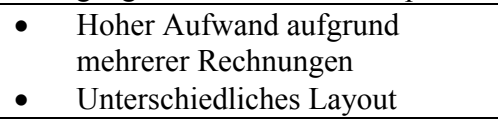 & $\begin{array}{ll}\text { - } & \text { Konsolidierte } \\
\text { Rechnungen }\end{array}$ \\
\hline $\begin{array}{l}\text { Leistung } \\
\text { bezahlen }\end{array}$ & - $\quad$ Mehrere Zahlungen & - $\quad$ Konsolidierte Zahlung \\
\hline $\begin{array}{l}\text { After-Sales- } \\
\text { Services }\end{array}$ & $\begin{array}{ll} & \text { Hoher Aufwand durch } \\
& \text { verschiedene Ansprechpartner }\end{array}$ & $\begin{array}{ll}- & \text { Kundenindividuelle und } \\
\text { zentrale Betreuung }\end{array}$ \\
\hline
\end{tabular}

Tabelle 3: Ineffizienzen im Beschaffungsprozess des Kunden und Gestaltungsmöglichkeiten für den Leistungsintegrator 
Tabelle 3 fasst exemplarisch Ineffizienzen im Kundenprozess und Gestaltungsmöglichkeiten in Form von Zusatzleistungen für den Leistungsintegrator zusammen. Dabei können die Zusatzleistungen je nach Kundensegment spezifisch sein. Die Aufgaben im Kundenprozess lehnen sich an das Customer Resource Life Cycle Modell von [IvLe84, S. 1196ff] an. Die Kundenprobleme und Zusatzleistungen sind aus den eingangs erwähnten Fallstudien abgeleitet worden.

Beispiel: Als Generalunternehmer koordiniert T-Systems International (TSI) verschiedene Leistungserbringer in Grossprojekten. Die Kunden erhielten in der Vergangenheit zahlreiche Einzelrechnungen der Leistungserbringer mit jeweils unterschiedlichem Layout. Im Sinne einer Kundenorientierung möchte TSI mittels konsolidierter Rechnungen die Transparenz über die erbrachten Leistungen erhöhen. Als Zusatzleistung werden die Rechnungen mit dem Ziel einer administrativen Vereinfachung individuell aufbereitet, z. B. durch Aufgliederung der Rechnungspositionen nach der Kostenstellenstruktur des Kunden. Voraussetzung ist eine zentrale Projektsteuerung und -abrechnung durch TSI sowie zentrale Rückmeldungen der erbrachten Leistungen durch die Leistungserbringer.

\subsection{Kooperationsprozesse in der Auftragsabwicklung}

Der Leistungsintegrator vernetzt sich mit seinen Lieferanten und Geschäftspartnern, um Zusatzleistungen für den Kunden zu erbringen. Die aufeinander abgestimmten Aktivitäten bilden dabei die Kooperationsprozesse. Sie spezifizieren die Aufgaben entlang einer Wertschöpfungskette zur Erstellung dieser Leistungen. Verschiedene Leistungen aus Tabelle 3 führen zu Abstimmungen im Geschäftsnetzwerk und zu kooperativen Teilprozessen. Einige Beispiele zeigt Tabelle 4, die aus den eingangs erwähnten Fallstudien abgeleitet wurden.

Beispiel: SIG Combibloc ist ein führender Anbieter von Verpackungslösungen für Getränke, Lebensmittel und Konsumgüter. Im Rahmen eines Pilotprojekts hat SIG Combibloc den Auftragsabwicklungsprozess umgestaltet [Seng03]. Der neue Prozess berücksichtigt gemeinsame Planungsaktivitäten mit dem Pilotkunden Coca Cola, der periodisch Bestands- und Bedarfsinformationen bereitstellt, anstatt wie zuvor in unregelmässigen Abständen Bestellungen zu erteilen. Auf Basis dieser Auftragsinformationen ermittelt SIG Combibloc die Liefermenge an Coca Cola. Da die Bestands- und Bedarfsinformationen allen internen Gesellschaften der SIG und den externen Lieferanten unmittelbar zur Verfügung stehen, können diese ihre Produktion frühzeitig ausrichten. 


\begin{tabular}{|l|l|}
\hline $\begin{array}{c}\text { Mikrokoopera- } \\
\text { tionsprozesse }\end{array}$ & \multicolumn{1}{|c|}{ Beschreibung } \\
\hline $\begin{array}{l}\text { Planung / } \\
\text { Auftragsauslösung }\end{array}$ & $\begin{array}{l}\text { Der Leistungsintegrator unterstützt den Kunden bei der Beschaf- } \\
\text { fungsplanung und der Bedarfsermittlung. Er übernimmt ggf. selbst } \\
\text { die Bestandsführung (Vendor Managed Inventory) auf Basis } \\
\text { periodisch aktualisierter Produktions- und Absatzpläne des Kunden. }\end{array}$ \\
\hline $\begin{array}{l}\text { Ermittlung der } \\
\text { Leistungs- } \\
\text { erbringer }\end{array}$ & $\begin{array}{l}\text { Der Leistungsintegrator ermittelt Partner für die Erfüllung des } \\
\text { Auftrags auf Basis gemeinsam festgelegter Regeln. Dabei können } \\
\text { bestehende Einkaufskontrakte oder übergreifende Verfügbarkeits- } \\
\text { und Kapazitätsprüfungen die Auswahl beeinflussen. Der direkte } \\
\text { Zugriff beispielsweise auf aktuelle Bestände oder Kapazitäten von } \\
\text { internen Geschäftseinheiten und externen Partnern erlaubt die } \\
\text { dynamische Ermittlung von Leistungserbringern. }\end{array}$ \\
\hline $\begin{array}{l}\text { Auftrags- } \\
\text { terminierung }\end{array}$ & $\begin{array}{l}\text { Der Leistungsintegrator sichert dem Kunden mittels einer } \\
\text { globalen“ Verfügbarkeitsprüfung Liefermengen und -termine je } \\
\text { Bestellposition zu. }\end{array}$ \\
\hline $\begin{array}{l}\text { Konsolidierte } \\
\text { Lieferung }\end{array}$ & $\begin{array}{l}\text { Der Leistungsintegrator steuert die Zusammenführung von Lieferun- } \\
\text { gen mit Logistikdienstleistern z. B. über Dispositions- oder } \\
\text { Montagepunkte [Chri98, S. 139ff]. }\end{array}$ \\
\hline $\begin{array}{l}\text { Kooperative } \\
\text { Rechnungs- } \\
\text { stellung }\end{array}$ & $\begin{array}{l}\text { Der Leistungsintegrator rechnet gegenüber dem Kunden die Ge- } \\
\text { samtleistung ab und verrechnet die Teilleistungen den Partnern } \\
\text { weiter. }\end{array}$ \\
\hline $\begin{array}{l}\text { Kooperatives } \\
\text { Beschwerde- } \\
\text { management }\end{array}$ & $\begin{array}{l}\text { Der Leistungsintegrator ordnet als zentraler Ansprechpartner des } \\
\text { Kunden die Reklamation dem auslösenden Kundenauftrag zu und } \\
\text { überträgt dem Kunden ggf. eine Gutschrift. Im Gegenzug belastet er } \\
\text { die Partner mittels einer Lastschrift. Beim Verschulden eines } \\
\text { Problems durch einen Partner kann dies in eine Statistik einfliessen, } \\
\text { um die Qualität des Prozesses zu verbessern. }\end{array}$ \\
\hline
\end{tabular}

Tabelle 4: Beispiele für kooperative Teilprozesse

\subsection{Managementprozesse in der Auftragsabwicklung}

Während Kunden- und Kooperationsprozesse die (bereitzustellenden) Leistungen festlegen, steuern die Managementprozesse die Ausführung von Kundenaufträgen mit dem Ziel der zuverlässigen Leistungserbringung für den Kunden. Dazu bedarf es eines konsequenten Prozessmanagements, das übergreifende Transparenz, Frühwarnmechanismen und übergreifende Auswertungen umfasst. Konsolidierte, zeitnahe Informationen stellen Transparenz über Planungsdaten, Auftrags-, Lieferund Transportstatus sowie Bestände her $\left[\operatorname{Kilg}^{+} 02\right]$. Voraussetzung sind Mappingund Clearingdienste zur Harmonisierung der Datensyntax und eine gemeinsame Semantik. Die Transparenz lässt sich dabei durch eine schnelle Informationsverfügbarkeit und hohe Datengenauigkeit verbessern. Aufbauend auf der Transparenz umfasst die Implementierung eines Frühwarnmechanismus zusätzlich die Definition von Ereignissen und Massnahmen [Niss02]. Ein hinterlegter Empfängerkreis erhält z. B. automatisch eine Nachricht bei „kritischen“ Ereignissen. Eine Analyse 
unterstützt ferner übergreifende Auswertungen, wie z. B. Leistungskennzahlen von Lieferanten und Transportdienstleistern [Bret ${ }^{+} 02$, S. 39].

Tabelle 5 stellt Objekte, Ereignisse, Massnahmen und Metriken für die kooperative Auftragsabwicklung beispielhaft dar.

\begin{tabular}{|c|c|}
\hline Managementprozesse & Beispiele \\
\hline $\begin{array}{l}\text { Transparenz } \\
\text { (Visibility) }\end{array}$ & \begin{tabular}{ll}
\multicolumn{2}{l}{ Objekte: } \\
$-\quad$ Aufträge (Order Visibility) \\
- & Lieferungen / Transporte (Delivery Visibility) \\
- & Bestände (Inventory Visibility) \\
- & Planungen (Planning Visibility) \\
\end{tabular} \\
\hline $\begin{array}{l}\text { Frühwarn- } \\
\text { mechanismus } \\
\text { (Supply Chain } \\
\text { Event Management) }\end{array}$ & $\begin{array}{ll}\text { Ereignisse / Reaktionen: } \\
\text { - } & \text { Fehler in Produktion / Austausch bzw. Rückrufaktion } \\
\text { - } & \text { Produktionsengpass / alternativen Lieferanten bestimmen } \\
\text { - } & \text { Transportengpass / Kalkulation der neuen Ankuftzeit } \\
\text { - } & \text { Überschreitung der Abholfrist / Versand mit einem } \\
& \text { alternativen Verkehrsmittel (z. B. Flugzeug) } \\
\text { - } & \text { Unterschreitung eines minimalen Bestandes (bei VMI) / } \\
& \text { Disponenten automatisiert informieren }\end{array}$ \\
\hline $\begin{array}{l}\text { Analyse } \\
\text { (Supply Chain } \\
\text { Reporting) }\end{array}$ & $\begin{array}{l}\text { Metriken: } \\
\text { Kosten: Lagerbestandskosten, Kapazitätsauslastung, } \\
\text { Anzahl von Anrufen bez. Statusabfragen im Call Center, } \\
\text { Personalkosten für die Erfassung von Aufträgen, etc. } \\
\text { Zeit: Durchlaufzeiten (Order-to-Deliver, Order-to-Cash), } \\
\text { Transportzeiten, Artikelsuchzeiten, etc. } \\
\text { Qualität: Fehlerhafte Lieferungen, Anzahl Retouren, } \\
\text { Verluste, Produktverfügbarkeit, Kapazitätsengpässe, } \\
\text { Auftragsdaten (Genauigkeit, Vollständigkeit, Konsistenz, } \\
\text { Einzigartigkeit, Aktualität), etc. }\end{array}$ \\
\hline
\end{tabular}

Tabelle 5: Ausprägungen für die kooperative Auftragsabwicklung (exemplarisch)

Beispiel: Für T-Systems International bedeutet Transparenz die Konsolidierung von verteilten Projektinformationen (Projektstatus) zur Etablierung eines übergreifenden Projektcontrollings. Ein Frühwarnmechanismus erlaubt die automatisierte (zeitnahe) Überwachung von Budget-, Plankosten- und Terminabweichungen. Eine Analyse erlaubt Rückschlüsse hinsichtlich des Projekts (z. B. Einhaltung der Verträge) und konsolidiert Erfahrungen für anschliessende Projekte.

\section{Systemarchitektur}

Die Systemarchitektur schafft die technischen und konzeptionellen Voraussetzungen für die Kooperation zwischen Unternehmen und entscheidet wesentlich mit über die Netzwerkfähigkeit. Dabei sind mehrere Schichten zu unterscheiden 
[Alt04, S. 182ff]. Die Applikationsarchitektur realisiert die unmittelbare Prozessunterstützung und besteht aus den Ebenen Präsentation, Funktionen und Daten. Die Integrationsarchitektur dient der Kopplung von Applikationen. Die Infrastrukturarchitektur bildet schliesslich mit Plattform- und Netzwerkkomponenten die Grundlage der Integrations- und Applikationsarchitektur. Dieses Kapitel zeigt typische Umsetzungsvarianten für die kooperative Auftragsabwicklung auf.

\subsection{Applikations- und Integrationsarchitektur}

Applikationsarchitekturen realisieren die Prozessunterstützung für die kooperative Auftragsabwicklung und umfassen hierfür Geschäftsapplikationen, wie z. B. ERP (Enterprise Resource Planning)-, CRM (Customer Relationship Management)-, SCM (Supply Chain Management)- oder SRM (Supplier Relationship Management)-Systeme, die jeweils spezifische Funktionalitäten bereitstellen.

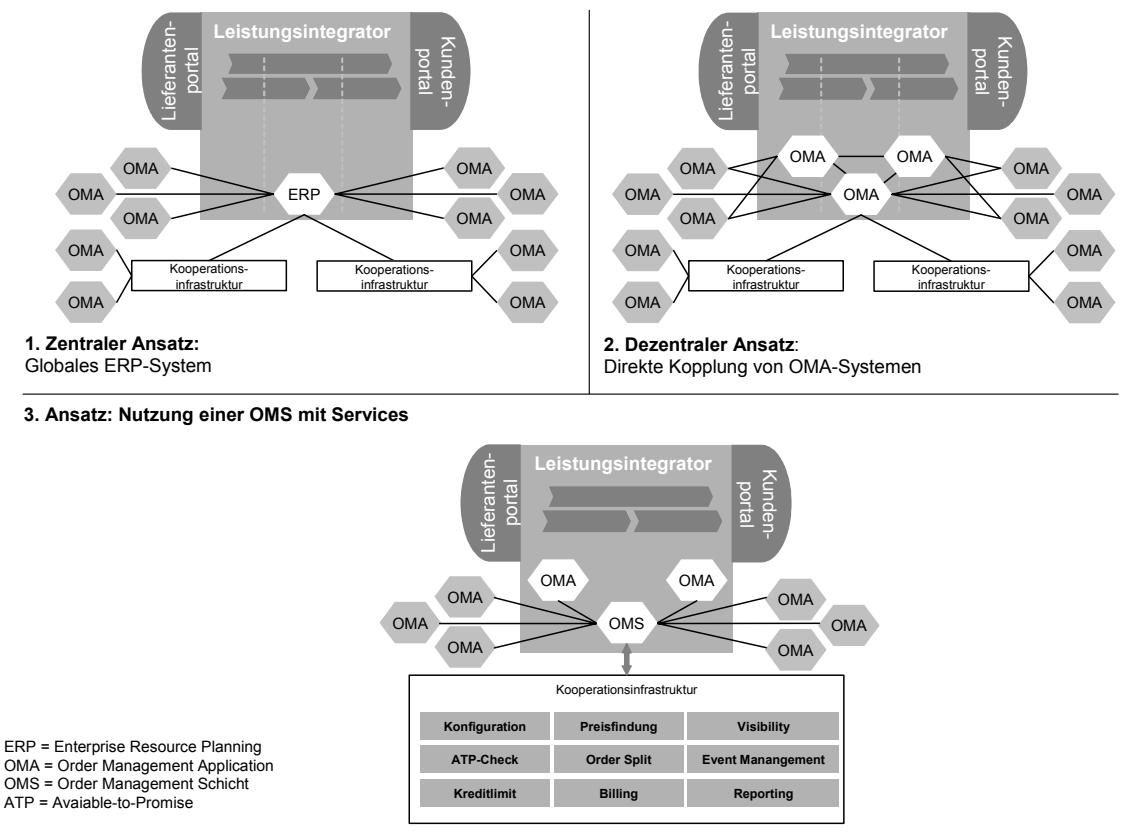

Abbildung 3: Übersicht der Umsetzungsalternativen

Integrationsarchitekturen regeln den durchgängigen Informationsfluss im Wertschöpfungsnetzwerk. ${ }^{2}$ Abbildung 3 stellt unterschiedliche Umsetzungsalternativen für die kooperative Auftragsabwicklung schematisch dar. Eine Geschäftsapplika-

2 Für eine ausführliche Diskussion der Applikationstypen und der Integrationsansätze für die kooperative Auftragsabwicklung siehe [GiLe04]. 
tion zur Unterstützung der Auftragsabwicklung wird dabei als Order Management Application (OMA) bezeichnet.

Für die Kopplung von Partnern und Kunden an die Systeme des Leistungsintegrators bestehen allgemein verschiedene Ansätze [Rieh97; Schu03]:

- Portale erlauben die Anbindung von Kunden und externen Partnern, die nicht in der Lage sind, einen elektronischen Datenaustausch zu etablieren, oder eine direkte Anbindung für nicht wirtschaftlich sinnvoll halten. Abbildung 3 stellt hierfür schematisch Kunden- und Lieferantenportale dar.

- Einen grösseren Automatisierungsgrad im Wertschöpfungsnetzwerk stellen bilaterale Kopplungen her. Hier gewinnt - in Nachfolge von EDI - die XMLbasierte Punkt-zu-Punkt-Integration an Bedeutung. In Abbildung 3 sind derartige Beziehungen durch Verbindungen der Systeme des Leistungsintegrators mit denen von Partnern und Kunden dargestellt.

- Weitere Möglichkeiten zur Vernetzung von Kunden und Partnern sind z. B. Exchanges (z. B. Elektronische Marktplätze), die wir als Kooperationsinfrastrukturen bzw. Business Collaboration Infrastrukturen (BCI) bezeichnen. Eine BCI ermöglicht die Zusammenarbeit zwischen mehreren Unternehmen und unterstützt viele-zu-viele (m:n) Beziehungen mit Mapping-, Sicherheitsund Transaktionsmanagementfunktionen. Dadurch werden Auftragsinformationen im Wertschöpfungsnetzwerk sofort verfügbar und können zur Koordination durch den Leistungsintegrator eingesetzt werden. Die Services der BCI unterstützen neben der Übermittlung, Validierung und Transformation von Geschäftsnachrichten auch einzelne Aufgaben, wie z. B. die Bestimmung von Partnern auf Basis von Auktionen, Zahlungs- oder Auftragsverfolgungsdienste.

Die Ansätze in Abbildung 3 unterscheiden sich im Wesentlichen in der Applikationslandschaft des Leistungsintegrators. Die folgenden Abschnitte bewerten die verschiedenen Umsetzungsmodelle.

\subsection{Globales ERP-System}

Ein Ansatz zur Umsetzung der kooperativen Auftragsabwicklung liegt für den Leistungsintegrator in der Nutzung eines globalen, unternehmensweit konsolidierten ERP-Systems [Huan04, S. 3]. Ein wichtiger Vorteil eines globalen Systems (1. Ansatz in Abbildung 3) ist, dass es Abläufe des Leistungsintegrators integriert, Transparenz und somit die Basis für eine ganzheitliche Auftragsabwicklung schafft.

Obwohl ERP-Systeme Funktionalitäten zur externen Vernetzung besitzen, ist eine übergreifende Prozesstransparenz und -kontrolle nicht per se gegeben [Newt01, S. 6]. Für die Umsetzung spezifischer Funktionen - wie z. B. die Zerlegung eines 
Kundenauftrags in einzelne Teilaufträge für Partner („Order Split“) auf Basis von Echtzeit-Informationen über Lagerbestände - sind Erweiterungen im ERP-System nötig [Huan04, S. 3]. Die Kosten für die Realisierung und Pflege solcher individuellen Lösungen sind sehr hoch. Die Nutzung eines ERP-Systems für die kooperative Auftragsabwicklung weist daher die in Tabelle 6 aufgeführten Schwachpunkte auf [LuBi00; Huan02, S. 4f; John03; WeSc03, S. 22ff]:

Schwachpunkte von ERP-Systemen für die kooperative Auftragsabwicklung

- $\quad$ Fokus liegt auf unternehmensinternen Prozessen

- $\quad$ Fehlende Funktionen zur unternehmensübergreifenden Steuerung von Kundenaufträgen (z. B."Order Split" auf Basis von Echtzeitinformationen)

- Beschränkte Standardfunktionen für die Integration von Statusinformationen externer Partner (z. B. Anstoss einer Rechnung auf Basis einer ASN bei Streckengeschäften)

- Fehlende Berücksichtigung von Prozessschritten mit externen Partnern (keine Unterstützung unternehmensübergreifender Workflows)

Tabelle 6: Schwachpunkte von ERP-Systemen für die kooperative Auftragsabwicklung

Nach einer Studie der Yankee Group, die mehr als 300 Entscheidungsträger befragt hat, sehen $40 \%$ der Befragten den Ausgangspunkt für die kooperative Auftragsabwicklung in einem globalen ERP-System [Huan04, S. 3]. Dieser Ansatz allein ist aufgrund der in Tabelle 6 aufgeführten Restriktionen aber nur dann wirtschaftlich sinnvoll, wenn wenige externe Partner integriert werden, der Koordinationsbedarf des Leistungsintegrators gering gehalten wird und kaum oder nur geringfügige Erweiterungen im ERP-System erforderlich sind.

Beispiel: myToys.de nutzt ein ERP-System für die kooperative Auftragsabwicklung. Verfügbarkeitsprüfungen im E-Shop werden erstmals bei der Übernahme eines Artikels in den Warenkorb durchgeführt. Da myToys.de einige Artikel nicht im eigenen Bestand führt, wird die Verfügbarkeit in der Regel direkt bei den Lieferanten angefragt [Müll04, S. 59ff]. Kann die Anfrage aber nicht performant ausgeführt werden, so greift myToys.de lokal auf Bestandsinformationen der Lieferanten zu, die es mehrmals am Tag von diesen bezieht.

\subsection{Direkte Integration von OMA-Systemen}

Der Ansatz eines globalen Systems für die kooperative Auftragsabwicklung ist für den Leistungsintegrator als Ausgangspunkt oft nicht möglich, da Unternehmen in der Regel mehrere OMA-Systeme zur automatisierten Steuerung der Auftragsabwicklung einsetzen (2. Ansatz in Abbildung 3). Eine von AMR durchgeführte Befragung von 400 Anwendern hat ergeben, dass Unternehmen abhängig von Produkten, Vertriebskanälen, Landesorganisationen und Kundensegmenten durchschnittlich 5,2 Systeme zur Auftragserfassung und 4,3 Systeme zur internen Auftragsabwicklung einsetzen [KeKr02, S. 2]. In solchen fragmentierten Applika- 
tionslandschaften bleiben Auftragsdaten selbst beim Leistungsintegrator verteilt. Die Verfügbarkeit von Echtzeitdaten über Auftragsstatus oder individualisierte Kundenansprachen („One-face-to-the-customer“) stellen unter diesen Bedingungen eine Herausforderung dar.

Die Umsetzung der kooperativen Auftragsabwicklung ist in einem solchem Umfeld aufgrund des Integrationsbedarfs mit einer hohen Komplexität verbunden. Im Vergleich zur Nutzung eines globalen Systems verstärken sich die Ineffizienzen: Voraussetzung für eine Durchgängigkeit und Transparenz ist die Kopplung verschiedener interner Auftragseingangs- und Ausführungssysteme. Die Zusammenarbeit mit externen Partnern wird zudem erschwert, da sie an mehreren Ausführungssystemen des Leistungsintegrators redundant gekoppelt sein können.

Neben einer bilateralen Punkt-zu-Punkt-Integration besteht EAI (Enterprise Application Integration) als ein Ansatz, um bestehende Applikationen des Leistungsintegrators zu koppeln [Holt03, S. 45f]. Trotz einer Vereinfachung der Schnittstellenkomplexität weist dieser Ansatz dieselben Nachteile wie der ERP-zentrierte Ansatz auf: Spezifische Funktionalitäten für die kooperative Auftragsabwicklung, wie etwa die dynamische Bestimmung von Partnern oder die kooperative Rechnungsabwicklung, müssen individuell implementiert werden.

Die direkte Integration von OMA-Systemen für die kooperative Auftragsabwicklung ist insgesamt mit hohen Implementierungskosten behaftet.

Beispiel: Die internen Gesellschaften der SIG Combibloc setzen jeweils ERPSysteme (SAP R/3) ein. Diese werden über ein EAI-Tool (Microsoft BizTalk Server) gekoppelt, welches eine durchgängige Auftragsabwicklung durch den Austausch von XML-Dateien ermöglicht. Die überbetriebliche Integration wird realisiert, indem Coca Cola die jeweiligen Daten zunächst im XML-Standard in einem vordefinierten Verzeichnis ablegt. Diese Verzeichnisse werden vom BizTalk Server periodisch eingelesen und mit der hinterlegten Verarbeitungslogik (Mappings, etc.) für die nachgelagerten Systeme der SIG-Gesellschaften aufbereitet. Die Bestellungen werden über den SAP Business Connector an die SAP R/3 Systeme der internen Gesellschaften weitergeleitet und automatisch angelegt. Die externen Lieferanten greifen über ein Lieferantenportal auf die Auftragsdaten zu.

\subsection{Nutzung einer Order Management Schicht mit Services}

Dieser Ansatz (3. Ansatz in Abbildung 3) versucht, die Nachteile der zuvor genannten Ansätze mittels einer zusätzlichen Serviceschicht aufzuheben. Eine zentrale Applikation (Order Management Schicht, OMS) enthält spezifische Funktionalitäten zur Ausführung und Steuerung des überbetrieblichen Auftragsdurchlaufs. Verschiedene Softwareanbieter (Escalate, i2, SAP oder Yantra) entwickeln entsprechende Standardlösungen für die kooperative Auftragsabwicklung und 
knüpfen damit an eine OMS an [Newt01]. Die Lösungen umfassen prozess-, prozessmanagement- und IS-spezifische Bausteine (s. Tabelle 7).

\begin{tabular}{|l|llll|}
\hline \multicolumn{1}{|c|}{ Ebenen } & \multicolumn{4}{c|}{ Bausteine der Standardlösungen } \\
\hline Prozess & $\bullet$ & $\begin{array}{l}\text { Koordination der } \\
\text { Transportabwicklung } \\
\text { Kooperative Rechnungsstellung }\end{array}$ & $\bullet$ & $\begin{array}{l}\text { Kooperatives } \\
\text { Beschwerdemanagement } \\
\text { Sourcing (Ermittlung } \\
\text { von Leistungserbringern) }\end{array}$ \\
\hline $\begin{array}{l}\text { Prozess- } \\
\text { management }\end{array}$ & $\bullet$ & $\begin{array}{l}\text { Übergreifende Transparenz } \\
\text { Übergreifendes Reporting }\end{array}$ & $\bullet$ & Frühwarnmechanismen \\
\hline System & $\bullet$ & $\begin{array}{l}\text { Diverse Auftragseingangskanäle } \\
\text { Werkzeuge für das Stammdaten- } \\
\text { management }\end{array}$ & $\bullet$ & Kopplungsmechanismen \\
\hline
\end{tabular}

Tabelle 7: Bausteine von Standardlösungen für die kooperative Auftragsabwicklung $\left[\mathrm{Giza}^{+}\right.$04, S. 85]

Charakteristisch für eine OMS ist, dass Funktionalitäten, wie z. B. die Auftragserfassung/-annahme oder die Rechnungsstellung zentralisiert werden. Die Vergabe von Auftragspositionen (Teilaufträgen) an externe Partner, konsolidierte Rechnungen oder die übergreifende Steuerung des Kundenauftrags lassen sich hierdurch beispielsweise effektiver realisieren. Abbildung 3 (3. Ansatz) gibt einen Überblick über verschiedene Services, die eine OMS bereitstellen kann.

Die Nutzung einer OMS verbessert insgesamt die Steuerung der übergreifenden Prozessabläufe, ermöglicht die Wiederverwendung implementierter Services und dadurch eine schnellere Konfiguration von kooperativen Auftragsabwicklungsprozessen. Der Nachteil liegt in den Kosten für den Aufbau einer OMS, die sich erst bei entsprechender Nutzung armotisieren.

Beispiel: Der ABB-Konzern entwickelt Lösungen für die Energie- und Automationstechnik und umfasst verschiedene Geschäftsbereiche mit jeweils weltweit verteilten Verkaufsorganisationen, Logistikzentren und Fabriken. ABB nutzt konzernweit Services für die Auftragsabwicklung („Global Order Management Services") mit dem Ziel, Logistikkosten zu senken und eine einheitliche Kundenansprache über die verschiedenen Verkaufsorganisationen zu realisieren [GiHe04]. Die Services schaffen eine übergreifende Transparenz über die im Konzern verteilten Bestände und realisieren eine zentrale, durchgängige Steuerung des Auftrags vom ersten Kundenkontakt über die Lieferung und Rechnungsabwicklung bis hin zur Retourenabwicklung. Da eine Punkt-zu-Punkt-Integration aller an der Auftragsabwicklung beteiligter Organisationseinheiten zu vielen komplexen Schnittstellen zwischen den verfügbaren ERP-Systemen geführt hätte, implementiert das Unternehmen eine OMS für die kooperative Auftragsabwicklung. Die Service-Schicht von ABB bietet z. B. Dienste für die Auftragserfassung, Verfügbarkeitsprüfung (für Endkunden und Verkaufsorganisationen), 
Lagerverwaltung und -optimierung (für Logistikzentren) oder Auftragsannahme, -bestätigung und Verrechnung (für Logistikzentren und Produktionswerke).

\section{Fazit und Ausblick}

Wettbewerbsvorteile, die Unternehmen in den letzten Jahren durch interne und durchgängige Prozesse erzielt haben, entstehen heute in Wertschöpfungsnetzwerken. Die kooperative Auftragsabwicklung stellt durchgängige Informationsflüsse über Unternehmensgrenzen hinaus her: Sie verhindert dadurch manuelle Wiedereingaben von Auftragsdaten, verbessert die organisationsübergreifenden Auftragsdurchlaufzeiten, eröffnet Potenziale zur Verbesserung der operativen Effizienz im Netzwerk und schafft damit die Basis für bessere Kundenbeziehungen. Eine engere Zusammenarbeit von Partnern zur Ausschöpfung dieser Potenziale hat jedoch einen höheren Koordinationsbedarf zur Folge.

Der Beitrag hat einen Architekturvorschlag für die kooperative Auftragsabwicklung entwickelt, der auf den drei Gestaltungsebenen $\mathrm{zu}$ folgenden Kernergebnissen geführt hat:

- Strategie. Ein Unternehmen nimmt innerhalb des Wertschöpfungsnetzwerkes die Rolle des „Orchestrators“ und somit des Leistungsintegrators ein. Dieser spezifiziert das Geschäftsmodell für die kooperative Auftragsabwicklung. Der Leistungsintegrator orientiert sich dabei an den Leistungen für seine Kunden und gestaltet entsprechend die Beziehungen $\mathrm{zu}$ den internen und externen Geschäftseinheiten.

- Prozess. Die Neuverteilung der Aufgaben in der kooperativen Auftragsabwicklung zwischen den Teilnehmern im Netzwerk geht von den beschaffungsbezogenen Prozessen der Kunden aus. Der Leistungsintegrator kann durch zusätzliche Leistungen seine Kunden binden, hat aber etwa für die Ermittlung der Leistungserbringer oder für die kooperative Rechnungsabwicklung einen höheren Koordinationsaufwand.

- System. Die integrierte Auftragsabwicklung stellt zusätzlich Anforderungen an die Informationssysteme des Leistungsintegrators. Da Unternehmen in der Regel mehrere Applikationen zur Unterstützung ihrer Auftragsabwicklung einsetzen, zeichnet sich die Nutzung einer zentralen Order Management Schicht mit Services als ein vielversprechender Ansatz zur Unterstützung der kooperativen Auftragsabwicklung ab. Entstehende Standardlösungen hierfür verbessern die technischen Voraussetzungen.

Leistungsintegratoren etablieren sich heute, wo Partner durch Ergänzung von Ressourcen und Fähigkeiten Wettbewerbsvorteile gewinnen (und somit die 
Bindung zum Kunden stärken) oder Effizienzpotenziale realisieren, die für alle Beteiligten eine Verbesserung ihrer Ausgangssituation bedeuten.

Auch wenn die Herausforderungen in der kooperativen Auftragsabwicklung durch spezifische Softwareprodukte relativiert werden können, bleiben dennoch zahlreiche organisatorische und technische Herausforderungen bestehen (s. Tabelle 8).

\begin{tabular}{|c|c|}
\hline Organisatorische Herausforderungen & Technische Herausforderungen \\
\hline $\begin{array}{l}\text { Koordination des Geschäftsnetzwerks: } \\
\text { Kein zentraler Zuständigkeitsbereich, } \\
\text { aber viele (zentrale) Koordinationsauf- } \\
\text { gaben durch übergreifende Abläufe } \\
\text { - } \quad \text { Misstrauen und mangelndes } \\
\text { Kooperationsverhalten zwischen } \\
\text { Geschäftsbereichen und/oder externen } \\
\text { Partnern (Austauschbarkeit) } \\
\text { - } \quad \text { Profit-Sharing } \\
\text { Change Management: Veränderungspro- } \\
\text { zesse benötigen viel Zeit für die } \\
\text { Umsetzung (Einnahme der } \\
\text { ganzheitlichen Sicht, Überwindung } \\
\text { bestehender Grenzen, etc.) }\end{array}$ & $\begin{array}{l}\text { - } \text { Schaffung von Applikations- und } \\
\text { Integrationsinfrastrukturen } \\
\text { - Standardisierung und } \\
\text { Harmonisierung der Stammdaten } \\
\text { und Nachrichtenformate im } \\
\text { Netzwerk } \\
\text { - Flexibilität der } \\
\text { Systemarchitekturen, um } \\
\text { wandelnde Kunden- und } \\
\text { Partnerpräferenzen schnell } \\
\text { umsetzen zu können }\end{array}$ \\
\hline
\end{tabular}

Tabelle 8: Herausforderungen in der kooperativen Auftragsabwicklung

\section{Literatur}

[Alt04] Alt, R.: Überbetriebliches Prozessmanagement. Habilitation, Universität St. Gallen, St. Gallen, 2004.

[Alt ${ }^{+}$04] Alt, R.; Gizanis, D.; Legner, C.: Collaborative Order Management: Toward Standard Solutions for Interorganizational Order Management. Erscheint in: Special Issue on International Journal of Technology Management, 2004.

[Bret ${ }^{+}$02] Bretzke, W.-R.; Stölzle, W.; Karrer, M.; Ploenes, P.: Vom Tracking \& Tracing zum Supply Chain Managment - aktueller Stand und Trends. Studie KPMG Consulting AG, Düsseldorf, 2002.

[BuZe04] Buck-Emden, R.; Zencke, P.: MySAP CRM - Kundenbezogene Geschäftsprozesse mit SAP CRM 4.0. Galileo Press: Bonn, 2004.

[ChKu00] Christiaanse, E.; Kumar, K.: ICT Enabled Co-ordination of Dynamic Supply Webs. In: International Journal of Physical Distribution and Logistics Management 30, 2000, S. 268-285.

[Chri98] Christopher, M.: Logistics and Supply Chain Management: Strategies for Reducing Costs and Improving Service. FinancialTimes / Pitman Pub: London, 1998.

[Flei01] Fleisch, E.: Das Netzwerkunternehmen. Springer, Berlin, etc., 2001. 
[GiHe04] Gizanis, D.; Heutschi, R.: Case study: Global Order Management Services Support Business at ABB. Arbeitsbericht der Universität St. Gallen, St. Gallen, 2004.

[GiLe04] Gizanis, D.; Legner, C.: Umsetzungsalternativen für die kooperative Auftragsabwicklung. In: Chamoni, P.; Deiters, W.; Gronau, N.; Kutsche, R.; Loos, P.; MüllerMerbach, H.; Rieger, B.; Sandkuhl, K. (Hrsg.): Multikonferenz Wirtschaftsinformatik (MKWI) 2004, Band 2. Akademische Verlagsgesellschaft Aka: Berlin, 2004, S. 63-75.

[Giza $\left.{ }^{+} 03\right]$ Gizanis, D.; Legner, C.; Grau, J.: Kooperative Auftragsabwicklung zwischen Handel und Konsumgüterindustrie. In: Uhr, W.; Esswein, W.; Schoop, E. (Hrsg.) Wirtschaftsinformatik 2003, Band I. Physica: Heidelberg, 2003, S. 531-552.

[Giza ${ }^{+}$04] Gizanis, D.; Alt, R.; Österle, H.; Gründel, K.; Reiss, T.: Logistik Web-Services in der kooperativen Auftragsabwicklung. In: Alt, R.; Österle, H. (Hrsg.) Real-time Business. Springer: Berlin, etc., 2004, S. 81-95.

[Heus99] Heuskel, D.: Wettbewerb jenseits von Industriegrenzen. Campus: Frankfurt, 1999.

[Hint03] Hinterhuber, A.: Value Chain Orchestration in Action and the Case of the Global Agrochemical Industry. In: Long Range Planning 35, 2002: S. 615-635.

[Holt03] Holten, R.: Integration von Informationssystemen. In: Wirtschaftsinformatik 45, 2003: S. 41-52.

[Huan02] Huang, K.: Unlocking the Potential for Distributed Order Management. Yankee Group Report, Boston (MA), 2002.

[Huan04] Huang, K.: Drivers For Distributed Order Management Reveals the Targets are Customer Service and Revenue Generation. Yankee Group Report, Boston (MA), 2004.

[IvLe84] Ives, B.; Learmonth, G. P.: The Information System as a Competitive Weapon. In: Communications of the ACM 27, 1984: S. 1193-1201.

[John03] Johnson, R.: Consolidated Order Management - ERP Alone Doesn`t Deliver. AMR Research, Report, 2003.

[KaKa00] Kakabadse, N.; Kakabadse, A.: Critical Review - Outsourcing: A Paradigm Shift. In: Journal of Management Development 19, 2000: S. 670-728.

[KeKr02] Keltz, H.; Kraus, B.: The State of Order Management Applications. AMR Research, Report, 2002.

[Kilg $\left.{ }^{+} 02\right]$ Kilgore, S. S.; Orlov, L. M.; Nakashima, T.: Grading Apps For Inventory and Order Visibility. Forrester Research, Report, 2002.

[Krae $\left.{ }^{+} 00\right]$ Kraemer, K. L.; Dedrick, J.; Yamashiro, S.: Refining and Extending the Business Model with Information Technology: Dell Computer Corporation. In: The Information Society 16, 2000: S. 5-21.

[KuHe03] Kuhn, A.; Hellingrath, B.: Auftragsmanagement in Netzwerken: Supply Chain Management. In: Bullinger, H.-J.; Warnecke, H.-J.; Westkämper, E. (Hrsg.) Neue Organisationsformen im Unternehmen. Springer: Berlin etc., 2003, S. 644-670. 
[LuBi00] Luttighuis, P. O.; Biemans, F.: ERP in the E-Commerce Era. Working Paper, 2000.

[Marb00] Marbacher, A.: Demand \& Supply Chain Management. Paul Haupt: Bern, 2000.

[Mätz96] Mätzke, M.: Strukturwandel in der Automobilindustrie und seine Arbeitsfolgen bei Zulieferern: Anmerkung zu problematischen Verallgemeinerung. In: SOFIMitteilungen 23, 1996: S. 67-80.

[Mert97] Mertens, P.: Integrierte Informationsverarbeitung, Band 1: Administrations- und Dispositionssysteme in der Industrie. Gabler: Wiesbaden, 1997.

[Mert ${ }^{+}$99] Mertens, P.; Faisst, W.; Zeier, A.: Rechnergestützte Koordination von Geschäftspartnern beim Auftragsdurchlauf. In: Faller, P. (Hrsg.) Transportwirtschaft im Umbruch. Linde: Wien, 1999, S. 353-361.

[Mül104] Müller-Wünsch, M.: Die eBusiness-IT-Architektur für Supply Chain Controlling und Supply Chain Operations am Beispiel des Retailkonzepts von myToys.de. In: Chamoni, P.; Deiters, W.; Gronau, N.; Kutsche, R.; Loos, P.; Müller-Merbach, H.; Rieger, B.; Sandkuhl, K. (Hrsg.) Multikonferenz Wirtschaftsinformatik (MKWI) 2004, Band 2. Akademische Verlagsgesellschaft Aka: Berlin, 2004, S. 52-62.

[Nerv02] NerveWire: Collaborative Commerce: Compelling Benefits, Significant Obstacles, Research Study. http://www.nervewire.com, Abruf am 2003-09-28.

[Newt01] Newton, C. J.: Managing Order Fulfillment Across the Supply Chain, AMR Research, Report, 2001.

[Niss02] Nissen, V.: Supply Chain Event Management. In: Wirtschaftsinformatik 44, 2002: S. 477-480.

[Nola97] Nolan, R. L.: Top-down Driven Architecture Design. In: Information Management \& Computer Security 5, 1997: S. 123-12.

[OlKa03] Oliva, R.; Kallenberg, R.: Managing the transition from products to services. In: International Journal of Service Industry Management 14, 2003: S. 160-172.

[Öste95] Österle, H.: Business Engineering: Prozess- und Systementwicklung. Band 1: Entwurfstechniken, 2. Auflage, Springer: Berlin etc., 1995.

[Otto04] Otto, A.: Auftragsabwicklung. In: Klaus, P.; Krieger, W. (Hrsg.) Gabler-Lexikon Logistik: Management logistischer Netzwerke und Flüsse. Gabler: Wiesbaden, 2004, S. 14-20.

[Rieh97] Riehm, R.: Integration von heterogenen Applikationen. Dissertation, Difo-Druck: Bamberg, 1997.

[Rock00] Rocks, D.: Dell's Second Web Revolution. In: Business Week 3699, 2000: S. EB62-64.

[Röhr $\left.{ }^{+} 00\right]$ Röhricht, J.; Teufel, T.; Willems, P.: SAP Prozesse - Vertrieb und Customer Service. Addison-Wesley: München, 2000.

[Sche92] Scheer, A.W.: Architektur integrierter Informationssysteme - Grundlagen der Unternehmensmodellierung. Springer: Heidelberg, 1992. 
[Sche02] Scheibler, J.: Vertrieb mit SAP - Prozesse, Funktionen, Szenarien. Galileo Press: Bonn, 2002.

[Schr02] Scherrik, S.: How Efficient Is that Company? In: Business Week 3813, 2002: S. 94-97.

[Schu03] Schubert, P.: E-Business-Integration, in: Schubert, P.; Wölfle, R.; Dettling, W. (Hrsg.) E-Business-Integration: Fallstudien zur Optimierung elektronischer Geschäftsprozesse. Hanser: München, 2003, S. 1-21.

[Seib90] Seibt, D.: Ausgewählte Probleme und Aufgaben der Wirtschaftsinformatik, In: Wirtschaftsinformatik 32, 1990: S. 7-19.

[Seng03] Senger, E.: Fallstudie: Supply Chain Prototyp für Coca-Cola Beverages und SIG Combibloc. Arbeitsbericht der Universität St. Gallen, St. Gallen, 2003.

[Sinz97] Sinz, E. J.: Architektur von Informationssystemen. In: Rechenberger, P.; Pomberger, G. (Hrsg.) Informatik-Handbuch. Hanser: München, 1997, S. 875-887.

[Stru97] Strunz, H.: Anwendungsarchitektur. In: Back, A.; Becker, J.; König, W.; Krallmann, H.; Mertens, P.; Rieger, B.; Scheer, A.-W.; Seibt, D.; Stahlknecht, P.; Thome, R.; Wedekind, H.; Strunz, H. (Hrsg.) Lexikon der Wirtschaftsinformatik. Springer: Berlin, 1997, S. 35-37.

[WeSc03] Wettklo, M.; Schultze, M.-A.: ERP-Strategien im collaborative Business. Detecon, 2003.

[WiBa99] Wise, R.; Baumgartner, P.: Go Downstream - The New Profit Imperative in Manufacturing. In: Harvard Business Review 77, 1999: S. 133-141.

[Zent $\left.{ }^{+} 04\right]$ Zentes, J.; Swoboda, B.; Morschett, D.: Internationales Wertschöpfungsmanagement. Vahlen: München, 2004. 